\title{
OR-75
}

\section{Kaempferia Parviflora wall. Ex Baker: A Source of PDE5 Inhibitors}

\author{
Prapapan Temkitthawon ${ }^{1, *}$, Thomas R. Hinds ${ }^{2}$, Joseph A. Beavo ${ }^{2}$, Jarupa Viyoch ${ }^{3}$, Khanit \\ Suwanborirux ${ }^{4}$, Pattara Sawasdee ${ }^{5}$ and Kornkanok Ingkaninan ${ }^{1}$
}

\begin{abstract}
${ }^{I}$ Department of Pharmaceutical Chemistry and Pharmacognosy and Center of Excellence for Innovation in Chemistry, Faculty of Pharmaceutical Sciences, Naresuan University, Phitsanulok 65000, Thailand; ${ }^{2}$ Department of Pharmacology, University of Washington, Seattle, USA; ${ }^{3}$ Department of Pharmaceutical Technology and Center of Excellence for Innovation in Chemistry, Faculty of Pharmaceutical Sciences, Naresuan University, Phitsanulok 65000, Thailand; ${ }^{4}$ Center for Bioactive Natural Products from Marine Organisms and Endophytic Fungi, Faculty of Pharmaceutical Sciences, Chulalongkorn University, Bangkok 10330, Thailand; ${ }^{5}$ Department of Chemistry, Faculty of Sciences, Chulalongkorn University, Bangkok 10330, Thailand; E-mail: prapapantem@gmail.com
\end{abstract}

Kaempferia parviflora Wall. ex Baker has been popularly used as male sexual performance enhancer in Thailand. However, its mechanisms were still unknown. One of the possible mechanism for this remedy is phosphodiesterase 5 (PDE5) inhibition. Among 41 plant extracts, the rhizomes of $K$. parviflora ethanolic extract could inhibit PDE5 at $50 \mu \mathrm{g} / \mathrm{ml}$. Therefore, eight 7 methoxyflavones which are the major the chemical constituents isolated from rhizomes of $K$. parviflora were further investigated on inhibitory activity against PDE5. Moreover, the selectivity of these compounds on PDE5 over PDE6 was evaluated. The results showed that 7-methoxyflavones from this plant showed inhibition effect toward both enzymes. The most potent PDE5 inhibitor was 5,7-dimethoxyflavone $\left(\mathrm{IC}_{50}=10.64 \pm 2.09 \mu \mathrm{M}\right.$, selectivity on PDE5 over PDE6 = 3.71). Structure activity relationship showed that the methoxyl group at C-5 position of 7-methoxyflavones was necessary for PDE5 inhibition. K. parviflora rhizome extract and its 7-methoxyflavone constituents had moderate inhibitory activity against PDE5. This finding provides an explanation for enhancing sexual performance in the traditional use of $K$. parviflora. Moreover, 5,7dimethoxyflavones could be a useful lead compound for clinically efficacious PDE5 inhibitors.

Keywords: Kaempferia parviflora, phosphodiesterase5, methoxyflavones, traditional sexual performance enhancer. 